\title{
De la revolución del software a la del hardware en educación superior
}

\section{From software to hardware revolution in higher education}

\author{
Carlos Castaño-Garrido \\ Urtza Garay-Ruiz \\ Universidad del País Vasco/Euskal Herriko Unibertsitatea, UPV/EHU (España)
}

Sotiris Themistokleous

Center for the Advancement of Research \& Development in Educational

Technology, CARDET, (Chipre)

\section{Resumen}

La revolución que supuso la democratización del software se amplía al hardware. Nos encontramos ante un nuevo escenario que incorpora hardware barato y de fácil acceso que posibilita el diseño y la creación de objetos reales. Ante esta nueva realidad, los contextos educativos deben reflexionar sobre su práctica, adaptarse y rediseñar sus espacios de aprendizaje tanto digitales como presenciales. En este trabajo repasamos las aportaciones que realiza el e-learning 2.0 a la educación en su conjunto. Presentamos un recorrido por las diversas y complejas oportunidades educativas que inciden en el desarrollo del blended learning y de las metodologías activas combinadas con tecnologías emergentes. Esto es, analizamos las aportaciones a la educación y la situación a la que instituciones educativas se enfrentan desde la revolución de la Web 2.o hasta los actuales contextos de aprendizaje híbridos y los espacios de creación o FabLabs que contribuyen a la difusión de la tenue frontera entre aprendizaje formal e informal. La evolución de la tecnología conlleva la adaptación de los procesos de aprendizaje a metodologías innovadoras para el desarrollo de las competencias en la resolución de problemas. En conclusión, diseñar contextos de aprendizaje basados en la horizontalidad de la transmisión y construcción del conocimiento para contribuir, de esta manera, al desarrollo de la propia tecnología.

Palabras clave: TIC (Tecnologías de la Información y la Comunicación); educación; competencia; universidad.

\begin{abstract}
The revolution in the democratization of software has reached hardware. We face a new scenario that incorporates cheap and easy to access hardware, which enables the design and creation of real objects. Faced with this new reality educational contexts must reflect on their practice and adapt to it by redesigning both their digital and face-to-face learning spaces. In
\end{abstract}


this paper, we review the contributions of eLearning 2.0 to education. We take a tour around the diverse and complex educational opportunities generated by the development of blended learning and the active methodologies combined with emerging technology. This is, we investigate the contributions of the Web 2.0 to education and the situation that educational institutions face from the Web 2.o revolution to the current hybrid learning contexts and the creation spaces or FabLabs that contribute to the diffusion of the fine line between formal and informal learning. The evolution of technology involves the adaptation of learning processes to innovative methodologies for the development of skills in problem solving. In conclusion, the design of learning contexts based on the horizontality of knowledge transmission and construction to contribute, in this way, to the development of technology itself.

Keywords: ICT (Information and Communication Technologies); education; competence; university.

El desarrollo tecnológico generado a raíz de la aparición en 2005 de la denominada Web 2.o (O’Reilly, 2005) ha traído consigo un nuevo escenario apenas reconocible hace unos pocos años. Un territorio definido por tres grandes avances. Por un lado, el auge y la rápida penetración en el mercado de una gran cantidad de dispositivos móviles que compiten con éxito en ventas y aceptación con los tradicionales ordenadores portátiles, que arrinconan a otros dispositivos recientes, como los netbooks, y que se caracterizan por su permanente conectividad.

En segundo lugar, la consolidación de la computación en la nube (cloud computing), como un nuevo modelo de prestación de servicios de negocio y tecnología, que nos permite el acceso a los servicios que utilizamos y a nuestros datos desde cualquier lugar, en cualquier momento y con cualquier tipo de dispositivo.

$\mathrm{Y}$, en tercer lugar, el desarrollo de un nuevo modelo de negocio (aplicaciones para estos dispositivos realizados por terceros, las denominadas "apps") aportando aplicaciones ligeras para ser utilizadas en los dispositivos actuales y que nos posibilitan ir más allá de las aplicaciones y de su utilización inicialmente prevista en teléfonos y tabletas.

Desde el punto de vista de la formación, estas tendencias posibilitan la eclosión de un nuevo movimiento basado en la ubicuidad y la conexión a la Red. Este escenario novedoso es ya una realidad. Tres datos para confirmarlo:

1. La existencia en el mercado universitario de cursos y experiencias educativas basadas en las metodologías derivadas de una manera innovadora de enfrentarse a la educación (Coursera, EdX, MiriadaX, etc.);

2. El movimiento de las universidades y organizaciones más prestigiosas de nuestro entorno hacia este nuevo territorio (Harvard, Oxford, el propio MIT); y

3. Su reconocimiento y visibilidad ya recogida en los informes más avanzados sobre tendencias en Tecnología y Educación (caso de los Informes Horizon, por ejemplo). 
Sin embargo, a veces da la impresión de que los desarrollos tecnológicos van más rápido que la capacidad tanto teórica como práctica de la educación para insertarlos en nuevos enfoques de aprendizaje y enseñanza (Castaño y Cabero, 2013). Y, sin embargo, todos estos desarrollos tecnológicos, para poder generar propuestas innovadoras y significativas, deben ser repensados desde la educación. Pero no con miedo al futuro sino con el espíritu de generar nuevos escenarios de formación basados en tecnología, de manera que sea posible tanto mejorar y actualizar la enseñanza tradicional universitaria, como acceder a nuevos yacimientos de población cada vez más significativos en la Sociedad del Conocimiento, como, por ejemplo, los ligados a la formación permanente de los profesionales (lifelong learning).

Es tiempo de recapitular y observar los nuevos desarrollos que la Web 2.o ha generado en contextos formativos, desde la primera revolución del software hasta la actual revolución del hardware, democratizando la tecnología en todos sus ámbitos para acercarnos al objetivo europeo de la construcción de la Sociedad del Conocimiento. Por ello, el objetivo de este artículo es doble. Por una parte, discutir el pretendido declive de la Web 2.o para defender la vigencia de sus aportaciones a la formación, elearning 2.0, y analizar sus tendencias, subrayando la potencialidad de los nuevos escenarios de aprendizaje híbridos junto con las nuevas metodologías de enseñanza-aprendizaje.

Y, en segundo lugar, conceptualizar estos entornos de aprendizaje híbridos con un nuevo concepto de blended learning que conjuga la potencialidad de la aportación clásica de la Web 2.0 con la revolución del hardware, configurando un nuevo movimiento. Definido como movimiento maker, se caracteriza por la utilización tanto de hardware barato y de fácil acceso, como de software de uso muy intuitivo y fácilmente disponible, para la fabricación de artefactos físicos. Están basados, por lo demás, en la cultura de compartir y colaborar (en línea o de forma presencial) y en el uso de estándares de diseño comunes para facilitar el intercambio entre los usuarios y la comunidad.

\section{LA WEB 2.0. LA REVOLUCIÓN DEL SOFTWARE Y LA ACTITUD DE LOS USUARIOS}

Se ha convertido en un tópico hablar del vertiginoso cambio de la tecnología, y, sin embargo, a veces no somos conscientes de su capacidad de constante cambio y actualización. Es cierto que pocas generaciones a lo largo de la historia tienen la oportunidad de ser testigos de cambios tecnológicos de la envergadura de aquellos a los que nos hemos enfrentado durante las dos últimas décadas, cambios que modifican los hábitos sociales y culturales y que se consideran como tecnologías disruptivas. En este sentido, es fácil reconocer que uno de ellos es la telefonía móvil, que ha revolucionado la forma en la que nos comunicamos. El otro, sin necesidad de pensarlo mucho, es Internet. Respecto a Internet, y desde el punto de vista 
educativo, pueden apuntarse dos grandes formas de utilización que han tenido una gran repercusión: el eLearning y la Web 2.0 (Llorente, 2012).

La Web 2.o entendida como un concepto que nace como contraposición a la web tradicional o, expresado con mayor propiedad, a los usos "tradicionales" de Internet (Castaño, Maiz, Palacio y Villarroel, 2008). Es importante remarcar esta idea: es un concepto, no un producto. Es una intención relacionada con la nueva manera de utilizar Internet. De una forma más personal, más participativa, más colaborativa. En palabras de O’Reilly (2015) la Web 2.0 es más una actitud que una tecnología.

Conviene recordar brevemente las principales características de la Web 2.0 según este autor, que pueden resumirse desde el punto de vista educativo en estas seis grandes ideas (Castaño et al., 2008):

- La web como plataforma. Que genera el surgimiento de múltiples nuevas aplicaciones dinámicas y colaborativas, además de ser herramientas sencillas e intuitivas.

- Cambios en la programación y composición web: separación entre diseño y contenido, utilización de estándares, interoperabilidad y diseño de software no limitado a un solo dispositivo.

- Concepción del software como servicio: servicios en línea y aplicaciones desde el propio navegador, interoperabilidad entre servicios y entre aplicaciones.

- Aprovechamiento de la inteligencia colectiva.

- Todos somos autores y podemos publicar: la Red de lectura y escritura, herramientas sencillas y potentes.

- Gestióndeloscontenidos:creaciónycomparticióndecontenidos,microcontenidos, utilización de metadatos, sindicación, etiquetado y folksonomía.

La revolución que esta nueva forma de entender la Red trae consigo en el mundo del software es de sobra conocida (Castaño, 2013). Pueden contarse por miles las aplicaciones Web 2.0 al servicio de los profesores y estudiantes. Estas aplicaciones pueden organizarse en cuatros grandes grupos: 1) Aplicaciones para compartir: 2) Herramientas para crear recursos; 3) Servicios para recuperar información; y 4) Redes Sociales.

La Web 2.o ha evolucionado hacia la denominada web semántica, sinónimo de la Web 3.o. Si la Web 2.o se centra en la participación, la Web 3.0 es acerca de los datos vinculados y la web semántica (Siemens, 2010). La Web 3.0 implica una experiencia web integrada, donde la máquina será capaz de comprender y catalogar los datos de una manera similar a los humanos. La idea es facilitar un almacén de datos mundial en el que cualquier formato de datos pueda ser compartido y comprendido por cualquier dispositivo en cualquier red (Rudman y Bruwer, 2016).

La evolución de la Web dará lugar a nuevas oportunidades y desafíos. Oportunidades derivadas de la integración autónoma de los datos y servicios, que aumentan las capacidades preexistentes de los servicios Web, así como la creación 
de nuevas funcionalidades. Los desafíos se refieren principalmente al acceso no autorizado y a la manipulación de datos, junto con el desarrollo de scripts y lenguajes dañinos. Estos desafíos han sido señalados igualmente por Tim Berners-Lee (2017) con ocasión del 28 cumpleaños de Internet.

\section{E-Learning 2.o. Nuevas posibilidades para la formación}

La pujanza del movimiento Web 2.o ha tenido un fuerte impacto en la educación, desarrollando nuevas perspectivas y acciones formativas soportadas en las redes telemáticas y generando el denominado e-Learning 2.o. Este tipo de aprendizaje, a diferencia del anterior al que podríamos denominar e-Learning 1.o, persigue, como señala Cabero (2013), que el estudiante adopte una actitud más activa y participativa en el proceso formativo, superando así el hecho de que el entorno tecnológico sea un simple repositorio de información puesto a disposición del estudiante y se convierta en un escenario de interacción y comunicación. La tabla 1 señala las diferencias fundamentales entre ambos ecosistemas en opinión de Sbnihi y Eddine (2010).

Tabla 1. Diferencias entre el e-Learning 1.0 y 2.0

\begin{tabular}{|l|l|}
\hline \multicolumn{1}{|c|}{ e-Learning 1.0 } & \multicolumn{1}{c|}{ e-Learning 2.0 } \\
\hline Plataforma LMS y CLMS & Herramientas de la Web 2.0 \\
\hline Basado en el profesor & Basado en el estudiante \\
\hline El profesor produce & El profesor valida \\
\hline El estudiante es espectador & El estudiante es productor \\
\hline Intercambio con la clase & Intercambio con la comunidad \\
\hline
\end{tabular}

Fuente: Sbnihi y Eddine (2010).

Este proceso de participación, colaboración y creación de recursos digitales por parte del alumno no sufrirá cambios en el proceso de evolución de la Web 2.o. Antes bien, las nuevas posibilidades de la web semántica parecen generar a su vez nuevos escenarios, a los que algunos denominan educación Web 3.0, que aumentan estas características señaladas. Sirva como ejemplo la aportación de Keats y Schmidt (2007) que se presenta en la tabla 2. 
Tabla 2. Generaciones de educación

\begin{tabular}{|c|c|c|c|}
\hline \multicolumn{4}{|c|}{ Generaciones de educación } \\
\hline Caracteristicas & Educación 1.0 & Educación 2.0 & Educación 3.0 \\
\hline $\begin{array}{l}\text { Función principal de } \\
\text { profesor }\end{array}$ & $\begin{array}{l}\text { Fuente de } \\
\text { conocimiento. }\end{array}$ & $\begin{array}{l}\text { Guía y fuente de } \\
\text { conocimiento. }\end{array}$ & $\begin{array}{l}\text { Guía en el proceso } \\
\text { de creación de } \\
\text { conocimiento } \\
\text { colaborativo. }\end{array}$ \\
\hline Tipo de contenido & $\begin{array}{l}\text { Materiales } \\
\text { tradicionales con } \\
\text { derechos de autor. }\end{array}$ & $\begin{array}{l}\text { Materiales con } \\
\text { derechos de autor y } \\
\text { recursos educativos } \\
\text { libres / abiertos. }\end{array}$ & $\begin{array}{l}\text { Recursos libres } \\
\text { abiertos / educativos } \\
\text { creados y reutilizados } \\
\text { por los estudiantes. }\end{array}$ \\
\hline $\begin{array}{l}\text { Actividades de } \\
\text { aprendizaje }\end{array}$ & $\begin{array}{l}\text { Tradicionales, } \\
\text { ensayos, tareas, } \\
\text { pruebas, algún } \\
\text { trabajo en grupo } \\
\text { dentro del aula. }\end{array}$ & $\begin{array}{l}\text { Los enfoques } \\
\text { tradicionales } \\
\text { transferidos } \\
\text { a tecnologías } \\
\text { más abiertas y } \\
\text { colaborativas. }\end{array}$ & $\begin{array}{l}\text { Actividades abiertas } \\
\text { y flexibles de } \\
\text { aprendizaje que } \\
\text { se centran en la } \\
\text { creación de espacios } \\
\text { para la creatividad } \\
\text { de los estudiantes; } \\
\text { redes sociales } \\
\text { fuera de los límites } \\
\text { institucionales. }\end{array}$ \\
\hline Rol institucional & $\begin{array}{l}\text { Límites fijos entre } \\
\text { las instituciones } \\
\text { basado en el } \\
\text { campus; enseñanza, } \\
\text { evaluación y } \\
\text { acreditación } \\
\text { proporcionada por } \\
\text { una institución. }\end{array}$ & $\begin{array}{l}\text { Aumento de la } \\
\text { colaboración entre } \\
\text { las universidades y } \\
\text { estudiantes. }\end{array}$ & $\begin{array}{l}\text { Afiliaciones } \\
\text { y relaciones } \\
\text { institucionales más } \\
\text { abiertas; entrada de } \\
\text { nuevas instituciones } \\
\text { que proporcionan } \\
\text { servicios de } \\
\text { educación superior. }\end{array}$ \\
\hline $\begin{array}{l}\text { El comportamiento } \\
\text { del estudiante }\end{array}$ & En gran parte pasivo. & $\begin{array}{l}\text { De pasivo a activo, } \\
\text { siendo responsable } \\
\text { de su propio } \\
\text { aprendizaje. }\end{array}$ & $\begin{array}{l}\text { Activo, responsable } \\
\text { de su aprendizaje. } \\
\text { Incluye la cocreación } \\
\text { de recursos y } \\
\text { oportunidades de } \\
\text { formación. }\end{array}$ \\
\hline Tecnología & $\begin{array}{l}\text { E-Learning a } \\
\text { través de LMS } \\
\text { institucionales. }\end{array}$ & $\begin{array}{l}\text { E-learning a través de } \\
\text { LMS institucionales } \\
\text { enriquecidos con la } \\
\text { de otras aplicaciones } \\
\text { Web } 2.0\end{array}$ & $\begin{array}{l}\text { Aprendizaje } \\
\text { electrónico } \\
\text { impulsado desde la } \\
\text { perspectiva de los } \\
\text { entornos distribuidos } \\
\text { de aprendizaje } \\
\text { personal de los } \\
\text { estudiantes. }\end{array}$ \\
\hline
\end{tabular}

Fuente: Adaptado de Keats y Schmidt (2007). 
Estas diferentes perspectivas deben modularse en función del lugar que ocupe nuestra propuesta formativa en el continuum de la enseñanza basada en tecnología. Bates (2015) identifica las siguientes modalidades de distribución en el aprendizaje online:

1. Enseñanza en clase sin tecnología en absoluto (lo que no es común en estos tiempos);

2. Aprendizaje semipresencial que implica una amplia variedad de diseños incluyendo:

- aprendizaje enriquecido con tecnología o tecnología como soporte de la clase (un ejemplo típico sería el uso de dispositivas de PowerPoint).

- sistemas de gestión del aprendizaje como soporte de la enseñanza presencial, como repositorio de los materiales de clase, de lecturas y tal vez de foros de discusión.

- grabación de clases para la clase invertida.

- un semestre en campus tipo residencial y dos semestres online (el modelo de la Royal Roads University).

- un tiempo corto presencial con actividades prácticas o formación precedida o seguida de un tiempo intensivo de estudio online.

3. Aprendizaje híbrido o flexible. Requiere el rediseño de la enseñanza de modo que los estudiantes puedan hacer la mayor parte del curso online y asistir a las clases presenciales solamente para actividades muy específicas como actividades de laboratorio y trabajos prácticos que no pueden realizarse online.

4. Aprendizaje totalmente online sin encuentros presenciales, que incluye:

- cursos de educación formal, que generalmente cubren el mismo contenido, competencias y evaluación que en la versión presencial.

- cursos de educación informal, como los de formación continua profesional, o cursos totalmente online, como los MOOC.

- recursos educativos abiertos, disponibles para descarga gratuita online, al que el instructor o los estudiantes pueden acceder como soporte del curso.

Los diversos desarrollos en la educación semipresencial "blended learning", al que también denominamos aprendizaje híbrido, merecen una mención especial ya que pueden aportar diversas posibilidades. Señalamos cuatro de ellas a continuación: 1) un replanteamiento mínimo de la enseñanza; 2) el rediseño de la enseñanza en el aula; 3) incorporar nuevos recursos de apoyo en el aula, o 4) rediseñar por completo la propuesta formativa. 
Las nuevas tendencias en materia de metodología didáctica (Edutrends, 2015, 2016), como el aprendizaje basado en proyectos, el aprendizaje invertido, el aprendizaje basado en retos y el aprendizaje flexible encuentran en estas distintas posibilidades de terreno abonado para la innovación educativa. Añadiríamos a estas posibilidades metodológicas la oportunidad del desarrollo gradual por parte del estudiante de las competencias tecnológicas y de aprendizaje independiente, claves para una sociedad digital.

\section{Actualidad del E-Learning 2.0}

Una mirada a Google Trends nos informará rápidamente de que las búsquedas relacionadas con la Web 2.0 van perdiendo interés entre los usuarios. Por otra parte, la búsqueda sobre Moodle, como ejemplo del LMS mejor situado en el mundo universitario, no solo se mantiene sino que cuadriplica al interés generado por los usuarios con respecto de la Web 2.0.

Cabe preguntarse si hemos traspasado ya lo que Gladwell (2010) denomina punto de inflexión (tipping point), (figura 1) y que se refiere a ese momento a partir del cual los efectos de un proyecto o idea se potencian al máximo creando un cambio significativo, hasta ponerse de moda y posteriormente convertirse en una tendencia. Y nos encontráramos ahora en un momento de declive del movimiento Web 2.o.

Figura 1. Punto de inflexión (tipping point) en la adquisición de un proyecto

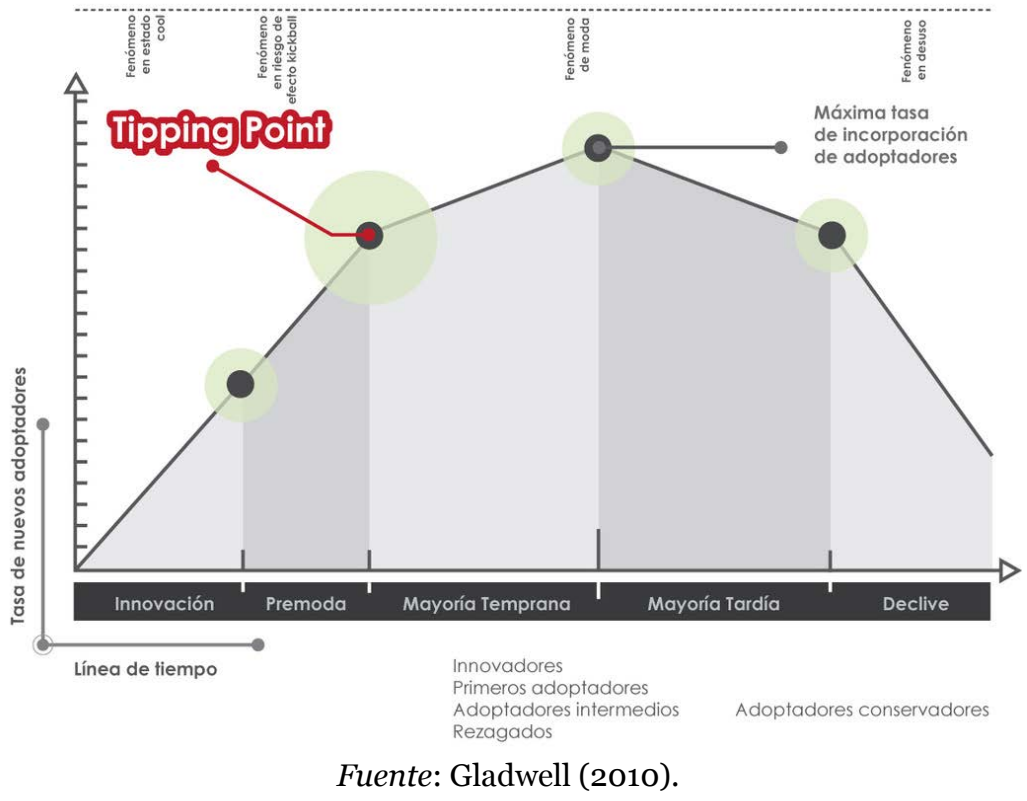


Sin embargo, hay elementos suficientes como para pensar que aún no hemos visto la eclosión del movimiento Web 2.o en la educación. Así, el informe presentado por Brown, Dehoney y Millichap (2015), publicado por Educause con patrocinio de la Fundación Bill y Melinda Gates, explora el salto que existe entre los LMS actuales y los entornos de aprendizaje susceptibles de cubrir las necesidades de la Educación Superior en materia digital. Y esto tanto en escenarios propiamente online como en el blended learning. En ambas situaciones tienden a utilizarse plataformas LMS o CLMS de manera mayoritaria. Una verdadera llamada a actualizar nuestros instrumentos tecnológicos.

Diferentes estudios señalan la más que notable implantación de este tipo de plataformas en la educación. Así, refiriéndose a las universidades norteamericanas, Dahlstrom, Brooks y Bichsel (2014), y Lang y Pirani (2014) indican que los LMS tienen presencia prácticamente en el $100 \%$ de las instituciones, y con más de un $85 \%$ de profesores usuarios de las mismas. Sin embargo, apenas alcanza al $41 \%$ el porcentaje de profesores que utilizan estas plataformas para promover la interacción fuera del aula de clase.

En nuestro contexto, y referido a la población escolar, Area (2012) en un informe de investigación realizado sobre la visión y opinión del profesorado sobre el Programa Escuela 2.0 en España, detecta una notable aceptación de la incorporación de las tecnologías Web 2.0 al aula, pero los efectos en la utilización de nuevos métodos y estrategias de enseñanza (metodología por proyectos, aprendizaje cooperativo, investigación en el aula, proyectos intercentros, etc.) son limitados.

En un momento en que la Educación Superior defiende alejarse del énfasis tradicional centrado en la figura del instructor, poniendo a los estudiantes en el centro del proceso, en que se están produciendo cambios en los cursos estándares presenciales, buscando experimentar con modelos diferentes (aprendizaje basado en problemas, aula invertida, etc.), los LMS tradicionales se encuentran en un momento comprometido.

En este sentido, veremos los futuros desarrollos de la era post LMS. Ya no es suficiente con actualizar y complementar los LMS con nuevas funcionalidades, sino que es necesario un nuevo acercamiento, capaz de responder a un modelo diferente de aprendizaje donde el modelo de transmisión de información está dejando paso a otro construido sobre conceptos tales como aprendizaje activo, personalización, curso híbrido y nuevas direcciones para medir el progreso de los aprendizajes (Brown, Dehoney y Millichap, 2015). Y, acaso haciendo un guiño a los pioneros de la Web 2.0 (Weinberger, 2005), proponen una nueva arquitectura "tipo Lego", donde las diferentes piezas forman parte de todo un conjunto.

Por lo tanto, la Web 2.o no ha alcanzado su punto de inflexión, permitiendo, en modalidades de blended learning ricas en tecnología, nuevos avances pedagógicos imposibles de realizar sin sus aplicaciones. Desde otro punto de vista, se detecta un fuerte movimiento universitario preocupado por la formación digital de sus 
estudiantes, que mira ya a una época post LMS basada en una arquitectura que fomente el aprendizaje activo y la colaboración entre estudiantes.

Pero aún hay más. Trabajo colaborativo no solamente en la construcción de conocimientos, sino incluso en la construcción de artefactos físicos, gracias a la nueva revolución del hardware. Revolución que sigue bebiendo de las fuentes de la Web 2.0 donde lo importante será, de nuevo, la actitud y el compromiso de los usuarios.

\section{LA REVOLUCIÓN DEL HARDWARE: CREAR, COMPARTIR Y APRENDER}

El informe Horizon 2016 para la Educación Superior describe seis tendencias clave y desafíos para la Educación Superior. Divididas en tres plazos, especifica respuestas y reacciones a esas tendencias y desafíos que determinarán el impacto de las seis herramientas y estrategias digitales en el ámbito de la Educación Superior: en un plazo corto ( 1 año o menos) lo que conocemos como BYOD o trae tu propio dispositivo y las tecnologías de aprendizaje adaptativo; en un plazo medio (2-3 años) la realidad aumentada y virtual y los makerspaces, y en un plazo largo (4-5 años) la computación afectiva y la robótica (Johnson, Adams, Cummins, Estrada, Freeman y Hall, 2016). Esta clasificación es directamente proporcional al nivel de dificultad de implantación de los desafíos a los que la universidad se enfrenta.

De este modo, con el objetivo de avanzar en una cultura del cambio y la innovación, en el informe se describen como retos sencillos para la enseñanza universitaria la mezcla entre el aprendizaje formal e informal y la mejora de alfabetización digital. Mientras que como difíciles encontramos los modelos de educación por competencias y la personalización del aprendizaje. Por tanto, como tendencia a corto plazo está la generalización de un tipo de aprendizaje mixto o híbrido, también conocido como blended learning, donde se conjuga espacio físico con virtual.

Una experiencia educativa cohesionada y flexible, y que permite conjugar un aprendizaje autónomo y colaborativo. Además, en los últimos años, han proliferado herramientas digitales que adoptan la forma de laboratorio virtual, entornos donde los estudiantes pueden realizar simulaciones y experimentos sin riesgo, y que facilitan el modelo de aprendizaje mixto basado en la clase invertida o flipped classroom (Johnson, Adams, Cummins, Estrada, Freeman y Hall, 2016).

Pero la universidad no puede quedarse solamente en el rediseño de sus entornos virtuales sino que debe replantearse también sus espacios físicos. La creación de entornos virtuales colaborativos, flexibles e innovadores acompañados de metodologías activas, debe ir de la mano de la creación de espacios físicos acordes a un aprendizaje más profundo y adaptado a las necesidades de la nueva realidad.

Esto es, en la Educación Superior, más allá de repensar los entornos virtuales, se debe replantear el diseño y la configuración de los espacios físicos de los entornos de aprendizaje. Para ello, es importante recordar que los estudiantes se implican más 
en espacios de aprendizaje flexibles, activos y colaboradores. West-Puckett (2014) señala que se pueden rediseñar los espacios físicos o aulas formales entendiendo el proceso de aprendizaje como integrador y conectado a través de proyectos, y no como conjunto de habilidades aisladas. Esta tendencia viene proyectada como mejora a medio plazo y sejustifica por la necesidad de indagar en el camino a un aprendizaje más profundo (Johnson et al., 2016). Buscar herramientas y metodologías de enseñanzaaprendizaje que proporcionen a los estudiantes habilidades para conseguir el éxito en un mundo laboral que se define como incierto e indefinido. Por ello, entre las competencias que debe desarrollar un estudiante destacan el pensamiento crítico y la competencia de aprender a aprender, o aprender a lo largo de la vida (Unesco, 2015).

El aprendizaje profundo se caracteriza porque el estudiante se centra en el significado del contenido, realiza conexiones profundas entre ideas y, además, las conecta con experiencias previas para potenciar su comprensión personal. En definitiva, un aprendizaje que despierta la curiosidad y las ganas de investigar de sus protagonistas (Johnson et al., 2016).

En este tipo de entorno de aprendizaje toman vida lo espacios denominados Makerspaces, FabLabs o las iniciativas Tinkering, englobados todos ellos en lo que se conoce como movimiento Maker. Este movimiento se basa en la filosofía DIY (Do-itYourself o Hazlo-tu-mismo). Rosenfeld y Sheridan (2014) señalan que ha generado mucho interés en el imaginario popular y decenas de miles de niños, jóvenes, adultos e incluso familias completas se sienten atraídos por él. Así la cultura del crear se ha convertido en una manera de expresar, y este impulso y sentimiento ha derivado en el surgimiento de espacios de creación digitales en diversos lugares, desde fábricas de galletas abandonadas a bibliotecas y desde escuelas a universidades. Por tanto, el movimiento maker engloba a un amplio y creciente número de personas que se dedican a la producción creativa y que utilizan foros físicos y digitales para compartir tanto el producto como el proceso. De ahí que se defina como una nueva revolución industrial (Anderson, 2012).

El movimiento maker se sustenta en tres pilares fundamentales: un objetivo común basado en actividades de diseño y construcción, espacios de creación que provienen de las comunidades de prácticas online y offline (Sheridan, Halverson, Brahms, Litts, Jacobs-Priebe y Owens, 2014; Wenger, 1998) y entender la creación como parte de la identidad del individuo (Dougherty, 2012). Por lo tanto, se caracteriza por el uso de herramientas digitales de escritorio, la cultura de compartir y colaborar en línea o de forma presencial, y el uso de estándares de diseño comunes para facilitar el intercambio (Martin, 2015; Rosenfeld y Sheridan, 2014).

La magnitud de su repercusión ha sido tal que actualmente ya existen varios manifiestos en relación a este movimiento tales como el Self Repair Manifiesto (https://es.ifixit.com/Manifesto), The Maker's Bill of Fights (https://makezine. com/2006/12/01/the-makers-bill-of-rights, o The Fixer's Manifiesto https:/ sugru.com/manifesto) (García, 2016a, p. 19). Entre todos ellos destaca el "Manifiesto 
del Movimiento Maker" propuesto por Hatch (2014), cofundador de TechShop, que es uno de los espacios de creación o makerspace más exitosos. En este documento inicial se presentan nueve ideas clave que cimientan el movimiento y que se resumen en los siguientes verbos de acción: hacer, compartir, dar, aprender, utilizar herramientas y tecnología, jugar, participar, apoyarse y cambiar (Hatch, 2014; Rosenfeld y Sheridan, 2014). El factor esencial del proceso es que el objetivo final sea la construcción colectiva de objetos físicos. Esto es, pasamos de la denominada revolución del software a la nueva revolución hardware. Una revolución que viene acompañada de la democratización de la tecnología mediante hardware barato y de fácil acceso para la fabricación digital. Además de software de uso muy intuitivo y disponible, lo que dota a la ciudadanía de herramientas computacionales poderosas para la fabricación de objetos tangibles (Anderson, 2012; Dougherty, 2012; Hatch, 2014).

Por lo tanto, la comunidad Maker no es solamente el uso de unas u otras herramientas como la impresión $3 \mathrm{D}$, la programación creativa con Arduino o wearables, sino una actitud ante el mundo. Un estado de ánimo que favorece el aprendizaje, la pasión por la ciencia y la confluencia de la interdisciplinaridad, desde las artes o el diseño hasta la tecnología de punta. Es aquello que pone en valor el poder de pensar, diseñar y crear de forma colectiva. Es decir, la cultural maker permite participar a todo el que esté interesado en el debate que definirá el futuro de la tecnología y sus aplicaciones sociales, éticas y económicas (Asry, 2016, p. 43). Por ello, proporciona nuevos entornos de transferencia del conocimiento más allá de lo formal y las instituciones tradicionales como las universidades o escuelas. Contribuye a la disolución de la frágil línea que diferencia el aprendizaje formal del informal y fomenta la creación de nuevas comunidades, tanto en línea como presenciales, que favorecen el desarrollo de la tecnología. Promueven, por tanto, la horizontalidad en la transmisión y creación del conocimiento.

De este modo, programas educativos basados en la experimentación han llegado a multitud de instituciones educativas del mundo. Esta tendencia, conocida como Maker Education, se enmarca dentro de los estudios STEAM (Science, Technology, Engireering, Arts, Mathematics) y ofrece una mezcla de herramientas, metodologías y tecnologías para hacer más activo y significativo el aprendizaje (García, 2016b, p. 36).

Prueba de su cada vez mayor introducción en el ámbito educativo son las diversas iniciativas educativas llevadas a cabo tanto a nivel internacional como nacional. Entre estas destacan FabAcademy del MIT, basado en el programa del curso "How to Make Almost Anything" (HT-MMA), dirigido por Gershenfeld y que dio origen a los FabLabs a los que se vinculan universidades y centros de investigación de prestigio como la Universidad de Michigan (García, 2016). FabLearn de la universidad de Stanford, cuyo objetivo es estudiar el impacto de los FabLabs en el aprendizaje. La publicación Make, especializada en este tipo de metodologías de aprendizaje y proyectos europeos de investigación y transferencia de conocimiento como 
FormaLab www.formalb.fr y Declic'in (declicin.info) (Martínez, 2016). Sin olvidar los diversos Maker Faire o ferias maker que se celebran en todo el mundo de forma periódica (ver makerfaire.com/map), donde destaca Maker Camp organizada por Make Media y Google. También destacan otro tipo de iniciativas como el grupo Clone Wars de la Universidad Carlos III de Madrid, proyecto referente en la construcción de comunidades de aprendizaje colectivo a nivel universitario, o los programas de fabricación digital de la Universidad Europea de Madrid y la Universidad Politécnica de Valencia.

Los espacios de construcción digital o espacios maker (FabLab, makerspace, etc.) en el ámbito educativo se sustentan en la filosofía "hazlo-tú-mismo", que no significa "hazlo-tú-solo". En los últimos años, incluso se empieza a hablar de DIWO, esto es, "Do-It-With-Other" o "Hazlo-con-otros". Procesos en los que se aplica a contextos grupales el concepto de "hazlo-tú-mismo". En este sentido destaca la iniciativa MasterDIWO (http://wiki.medialab-prado.es/index.php/Master DIWO (García, 2016a, p. 30).

No obstante, el objetivo final no es solamente crear, sino construir nuevas dinámicas basadas en el desarrollo de competencias mediante la realización de proyectos colectivos acompañados de tecnología, fomentando que se genere investigación empírica creíble por parte de los estudiantes (Asry, 2016, p. 48). Aspectos que ayudan al desarrollo de las competencias aprender a aprender y aprender a lo largo de la vida, que están directamente relacionadas con la concepción de que el diseño de contextos educativos para el aprendizaje no debe estar centrado únicamente en las herramientas, sino en el proceso y el producto.

Por lo tanto, la implantación de este tipo de espacios para la construcción en el contexto universitario conlleva seguir con un movimiento de ruptura entre los aprendizajes formales e informales, que viene íntimamente ligado al concepto de aprendizaje ubicuo (Zapata-Ros, 2015). Los FabLabs son talleres informales que se desarrollan en las instalaciones de una comunidad educativa conjugando lo físico con lo digital y donde se ofrece un acceso comunitario y cooperativo a dispositivos y suministros para la creación y consecución de un prototipo o producto (Johnson et al., 2016; Martín, 2015). Su objetivo principal es que los estudiantes se impliquen en la resolución creativa de problemas y desarrollen un pensamiento complejo. Lo que conlleva el desarrollo del ingenio y el pensamiento creativo y crítico ante los problemas o retos a los que se enfrenta.

Se trata de establecer nuevas dinámicas para la adquisición de competencias básicas mediante la realización de proyectos (Schneider, Krajcik, Marx y Soloway, 2002) como eje central del proceso de enseñanza-aprendizaje. Fomentar un aprendizaje significativo basado en el aprendizaje entre pares (DiGiacomo y Gutiérrez, 2015; Fleming, 2015) y fundamentado en la construcción de un producto final y la consecución de redes para el aprendizaje y la exposición pública del producto, todo ello mediado por el uso de las nuevas tendencias tecnológicas. 
Encontramos, por tanto, que Los FabLab para el ámbito académico, que se crearon por el Instituto de Tecnología de Massachusetss (MIT) (Gershenfed, 2005), son entornos pedagógicos que permiten a los estudiantes resolver problemas produciendo sus propias herramientas mediante el uso combinado de herramientas digitales y físicas. De este modo, se puede señalar que estos espacios de creación se fundamentan en tres teorías del aprendizaje: el conductismo, los alumnos asimilarán contenidos aprendiendo a partir de fuentes pre existentes. El constructivismo, se desarrollarán nuevos esquemas mentales que permitan resolver problemas y retos. En lugar de asumir que existe una solución, se priorizará el descubrimiento y la construcción de soluciones, esto es, aprender mediante la construcción del conocimiento (Martínez y Stager, 2013; Papert, 1991). Por último, el conectivismo, postulando que el aprendizaje se produce a través de la interacción con otras personas, tejiendo redes para abarcar nuevos retos y enfatizando la toma de decisiones (García b, 2016; Siemens, 2004).

En resumen, la creación de los espacios FabLab son un ecosistema de creación fértil, que promueven el aprendizaje significativo entre pares y la construcción y transferencia del conocimiento por parte de todos sus participantes. Por ello, estos nuevos escenarios que se vislumbran en un espacio breve de tiempo, nos deben empujar a reflexionar de forma profunda sobre cómo y dónde ocurre el aprendizaje, esto es, quién, qué y cómo aprender en la universidad del siglo XXI (Rosenfeld y Sheridan, 2014).

\section{CONCLUSIÓN}

El desarrollo tecnológico ha contribuido a la eclosión de tendencias educativas basadas en un nuevo movimiento cimentado en la ubicuidad y en Internet. La Web 2.o ha traído consigo una nueva forma de entender la red y la democratización del mundo del software (Castaño, 2013). Esto contribuye a un cambio de actitud del estudiante ante su propio aprendizaje. De ser pasivo pasa a ser activo y participativo en el proceso. Además, los entornos tecnológicos de aprendizaje se convierten en escenarios de interacción y comunicación, más allá de los repositorios estáticos.

En esta vorágine destacan los diferentes desarrollos dela educación semipresencial o blended learning que proporcionan diversas posibilidades educativas. Con ellas comienzan a difuminarse las barreras entre el aprendizaje formal e informal y se rediseñan tanto las propuestas formativas, como las metodologías y las propias aulas físicas. Se vislumbran nuevas metodologías didácticas basadas en el aprendizaje por proyectos o el aula invertida, lo que conlleva permitir escenarios para sembrar una innovación educativa que posibilite el desarrollo de las competencias del alumnado para una sociedad digital como la nuestra.

Nos encontramos por tanto en la era post LMS, donde el modelo de transmisión es horizontal (Brown, Dhonwy y Millichap, 2015) y la construcción del conocimiento se hace de forma colectiva. Actualmente podemos encontrar miles de aplicaciones 
Web 2.o al servicio de los agentes educativos. En cambio, parece que la Web 2.0 no ha alcanzado su punto de inflexión en la educación, lo que permite, todavía, nuevos avances pedagógicos basados en modalidades de enseñanza blended learning o entornos de aprendizaje híbridos. Una formación digital de los estudiantes universitarios que mira a una nueva época que fomenta el aprendizaje activo y basado en la creación y la colaboración (Cabero, 2013).

La revolución del software ha dado paso a una nueva revolución del hardware, lo que necesariamente viene acompañado del rediseño de los contextos de aprendizaje digitales y físicos de las instituciones educativas y universidades (West-Puckett, 2014). Además, es necesaria la adaptación de las metodologías de aprendizaje y caminar hacia nuevas formas de enseñar y aprender basadas en el diseño y construcción colaborativa de proyectos, e incluso artefactos físicos. Un cambio que sigue bebiendo de las fuentes de la actitud Web 2.0, donde el compromiso y la colaboración son el eje fundamental para el desarrollo de las competencias de aprender a aprender y el aprendizaje a lo largo de la vida. En definitiva, el futuro del aprendizaje mediado por la tecnología pasa por diseñar contextos de aprendizaje basados en la horizontalidad de la trasmisión y construcción del conocimiento lo que contribuye, al mismo tiempo, al desarrollo de la propia tecnología.

\section{APOYOS}

Proyecto de investigación financiado por el Grupo de Investigación Consolidado del Sistema Universitario Vasco, con referencia IT929-16.

\section{REFERENCIAS BIBLIOGRÁFICAS}

Anderson, C. (2012). Makers: The new industrial revolution. Nueva York: Crown.

Area, M. (Dir.) (2012). La visión y opinión del profesorado sobre el Programa Escuela 2.o en España Un análisis por comunidades autónomas. Proyecto de investigación "Las políticas de un «ordenador por niño» en España. Visiones y prácticas del profesorado ante el programa escuela 2.o. Un análisis comparado entre comunidades autónomas". Recuperado de http:/ tecedu.webs.ull.es/ticse20/media/files/ InformeFinal\%20Escuela20-Prof2012. pdf

Arsy, K. (2016). Entornos de aprendizaje en una Antigua Fábrica de Galletas.
El caso del Espacio Open en Bilbao. Comunicación y Pedagogía, 291-292, 4248.

Bates, A. W. (2015). La Enseñanza en la Era Digital. Una guía para la enseñanza y el aprendizaje. BC Campus. Recuperado de https://open.bccampus.ca/find-opentextbooks/?uuid=da5of5f1-bbc6-481ea359-e73007c66932

Berners-Lee, T. (2017). Three challenges for the web, according to its inventor. World Wide Web Foundation. Recuperado de https://webfoundation.org/2017/03/ web-turns-28-letter/

Brown, M., Dehoney, J., y Millichap, Nancy (2015). The Next Generation Digital Learning Environment. A Report on 
Research. Educause Learning Initiative. Recuperado de https://library.educause. edu/resources/2015/4/the-nextgeneration-digital-learning-environmenta-report-on-research

Cabero, C. (2013). La formación virtual en el entramado 2.0: el elearning 2.0. En J. Aguaded y J. Cabero (Coords.), Tecnologías y medios para la educación en la e-sociedad, 23-51. Madrid: Alianza Editorial.

Castaño, C., y Cabero, J. (2013). Enseñar $y$ aprender en entornos m-learning. Madrid: Síntesis.

Castaño, C., Maíz, I., Palacio, G., y Villarroel, J. D. (2008). Prácticas educativas en entornos Web 2.o. Madrid: Síntesis.

Castaño, C. (2013). Tecnologías y medios para la educación en la e-sociedad. Los recursos de la Web 2.0: su utilización educativa. En J. Aguaded y J. Cabero (Coords.), Tecnologías y medios para la educación en la e-sociedad, 53-69. Madrid: Alianza Editorial.

Dahlstrom, E., Brooks, D. Ch., y Bichsel, J. (2014). The Current Ecosystem of Learning Management Systems in Higher Education: Student, Faculty, and IT Perspectives. Louisville, CO: Educause Center for Analysis and Research. Research report. Recuperado de https:// library.educause.edu/resources/2014/9/ the-current-ecosystem-of-learningmanagement-systems-in-highereducation-student-faculty-and-itperspectives

DiGiacomo, D. K., y Gutiérrez, K. D. (2015). Relational Equity as a Design Tool Within Making and Tinkering Activities. Mind, Culture and activity, 23(2), 141-153.

Dougherty, D. (2012). The maker movement. Innovations, 7(3), 11-14.

Edutrends (2015). Radar de innovación educativa. Monterrey, México: Instituto Tecnológico de Monterrey. Recuperado de https://observatorio.itesm.mx/ edutrendsradar2015
Edutrends (2016). Radar de innovación educativa de preparatoria. Monterrey, México: Instituto Tecnológico de Monterrey. Recuperado de https: //observatorio.itesm.mx/ edutrendsradarpreparatoria2016

Fleming, L. (2015). Worlds of Making: Best Practices for Establishing a Makerspace for Your School. Londres: Corwin.

García Sáez, C. (2016a). (Casi) Todo por hacer. Una mirada social y educativa sobre los Fab Labs y el movimiento maker. España: Fundación Orange. Recuperado de http://www.fundacionorange.es/ wp-content/uploads/2016/05/Estudio Fablabs Casi Todo por hacer.pdf

García Sáez, C. (2016b). Educación en entornos Maker. Comunicación $y$ Pedagogía, 291-292, 36-41.

Gladwell, M. (2010). The tipping point. How Little things can make a big difference. Nueva York: Abacus.

Gershenfed, A. (2005). Fab: The coming revolution on your desktop from personal computers to personal fabrication. Nueva York: Peter Lane.

Hatch, M. (2014). The maker movement manifiesto. Nueva York: McGraw-Hill.

Johnson, L., Adams Becker, S., Cummins, M., Estrada, V., Freeman, A., y Hall, C. (2016). NMC Informe Horizon 2016 Edición Superior de Educación. Austin, Texas: The New Media Consortium.

Keats, D. W., y Schmidt, J. (2007). The genesis and emergence of Education 3.0 in higher education and its potential for Africa. First Monday, 12(3) Recuperado de http://firstmonday.org/issues/ issue12 3 /keats/index.html

Leah-Lang, L., y Pirani, J. A. (2014). The Learning Management System Evolution: CDS Spotlight Report, research bulletin. Louisville, CO: Educause Center for Analysis and Research. Recuperado de https://library. educause.edu/resources $/ 2014 / 5 /$ the learning-management-system-evolution 
Llorente Cejudo, M. C. (2012). El e-learning 2.0: de la tecnología a la metodología. @ tic. Revista d'innovació educativa, 9, 7986.

Martin, L. (2015). The Promise of the Maker Movement for Education, Journal of PreCollege Engineering Education Research (J-PEER), 5(1), 30-39.

Martínez, O. (2016). MakerConvent: espacio de aprendizaje STEAM. Comunicación y Pedagogía, 291-292, 49-53.

Martínez, S. L., y Stager, G. S. (2013). Invent to learn: Making, tinkering, and engineering in the classroom. Santa Bárbara, CA: Constructing modern Knowledge press.

O'Reilly, T. (2005). What is Web 2.0. Design Patterns and Business Models for the Next Generation of Software. Recuperado de http://www.oreilly.com/pub/a/web2/ archive/what-is-web-20.htm

Papert, S. (1991). Situation constructionism. En: S. Papert e I. Harel (Eds.), Constructionism 335-364. Cambridge: MIT Press.

Rosenfeld, E., y Sheridan, K. (2014). The Maker Movement in Education. Harvard Educational Review, 88(4), 495-504, doi: 10.17763/haer.84.4.34j1g68140382063

Rudman, R., y Bruwer, R (2016). Defining Web 3.0: opportunities and challenges. The Electronic Library, 34(1), 132-154. doi: 10.1108/EL-08-2014-0140.

Sbnihi, B., y Eddine, K. (2010). Towards a participatory e-learning 2.o. International Journal on Computer Science and Engineering, 2(1), 1-7.

Schneider, R., Krajcik, J., Marx, R. W., y Soloway, E. (2002). Student learning in project based science classroom. Journal of Research in Science Teaching, 39(5), 410-422.
Sheridan, K. M., Halverson, E. R., Litts, B. K., Brahms, L., Jacobs-Priebe, L., y Owens, T. (2014). Learning in the making: A comparative case study of three makerspaces. Harvard Educational Review, 84(4), 505-531.

Siemens, G. (2004). Conectivismo: Una teoría de aprendizaje para la era digital. Recuperado de www.fce.ues. edu.sv/uploads/pdf/siemens-2004conectivismo.pdf

Siemens, G. (2010). Web 3.0 / xWeb. Elearnspace. Recuperado de www. elearnspace.org/blog/2010/09/30/web3-oxweb/

Weinberger, D. (2005). Small pieces loosey joined. Recuperado de http://www. smallpieces.com/

UNESCO, (2015). Rethinking Education: Towards a global common good? Francia: Organización de las Naciones Unidas para la Educación, la Ciencia y la Cultura. Recuperado de http://unesdoc.unesco. org/images/o023/002325/232555e.pdf

Wenger, E. (1998). Communities of practice: Learning, meaning and identity. Cambridge: Cambridge University Press.

West-Puckett, S. (2014). Remaking education: Designing classroom markerspaces for transformative learning. Edutopia. Recuperado de https:/ www.edutopia.org/blog/classroommakerspaces-transformative-learningstephanie-west-puckett

Zapata-Ros, M. (2015). Teorías y modelos sobre el aprendizaje en entornos conectados y ubicuos, EKS, 16(1), 69102. doi: http://dx.doi.org/10.14201 esk1516169102 


\section{PERFIL ACADÉMICO Y PROFESIONAL DE LOS AUTORES}

Carlos Castaño Garrido. Profesor titular de la Universidad del País Vasco (UPV/EHU), Doctor en Pedagogía. Experto en Tecnología Educativa y director del Máster Oficial en Tecnología, Aprendizaje y Educación. Director del grupo de investigación consolidado del Sistema Vasco de Investigación "Weblearner". Líneas de investigación: MOOC, Realidad Aumentada, Web 2.o, e-Learning y Formación de Profesores.

E-mail: carlos.castano@ehu.eus

Urtza Garay Ruiz. Profesora y Directora de Innovación Metodológica de la UPV/EHU. Doctora en Pedagogía. Profesora del Máster Oficial en Tecnología, Aprendizaje y Educación y miembro del grupo de investigación consolidado del Sistema Vasco de Investigación "Weblearner". Líneas de investigación: MOOC, Realidad Aumentada, PLE, Plurilingüismo y Formación del profesorado.

E-mail: urtza.garay@ehu.eus

\section{DIRECCIÓN DE LOS AUTORES}

Escuela de Magisterio de Bilbao

Universidad del País Vasco/Euskal Herriko Unibertsitaea

Barrio Sarriena $\mathrm{s} / \mathrm{n}$

Código Postal 48940

Leioa, Bizkaia (España)

Sotiris Themistokleous. Director adjunto de CARDET, Centro Investigación para el desarrollo de la Tecnología Educativa de Nicosia, Chipre. Es doctor por la Universidad de Nicosia y ha trabajado en el American College of Thessaloniki, Greek State Archives y en el Mediterranean Institute of Gener Studies. Coordina el Proyecto Europeo de Investigación "Online Game for Civic Engagement of EU Citizens (ENGAME)". Sus líneas de trabajo son la formación del profesorado y la tecnología aplicada a la educación.

E-mail: sotiris.t@cardet.org

\section{DIRECCIÓN DEL AUTOR}

\section{CARDET}

Lykavitou Avenue 29, 1st floor

Código Postal 2401

Nicosia (Chipre) 
Fecha de recepción del artículo: 18/04/2017

Fecha de aceptación del artículo: 30/06/2017

\section{Como citar este artículo:}

Castaño Garrido, C., Garay Ruiz, U., y Themistokleous, S. (2018). De la revolución del software a la del hardware en educación superior. RIED. Revista Iberoamericana de Educación a Distancia, 21(1), pp. 135-153. doi: http://dx.doi. org/10.5944/ried.21.1.18823 